\title{
Ecological Relationship between Mesothelioma Incidence/Mortality and Asbestos Consumption in Ten Western Countries and Japan
}

\author{
Ken Takahashi ${ }^{1}$, Matti S Huuskonen ${ }^{2}$, Antti Tossavainen ${ }^{2}$, Toshiaki Higashi ${ }^{1}$, \\ Toshiteru OKUBO' ${ }^{1}$ and Jorma RanTANEN ${ }^{2}$ \\ 'Institute of Industrial Ecological Sciences, University of Occupational and Environmental Health, \\ ${ }^{2}$ Finnish Institute of Occupational Health, Helsinki, Finland
}

\begin{abstract}
Ecological Relationship between Mesothelioma Incidence/Mortality and Asbestos Consumption in Ten Western Countries and Japan: Ken TAKaHASHI, et al. Institute of Industrial Ecological Sciences, University of Occupational and Environmental Health-The objective of the present study was to evaluate the ecological relationship between mesothelioma incidence/mortality and per capita asbestos consumption in ten Western countries and Japan. The two national indices used to assess the geographical correlation were the most recent incidence/mortality rate of mesothelioma for the population over 15 years of age, and the per capita asbestos consumption rate of approximately 10-25 years ago for the population of all ages at that time. Among the ten Western countries, a clear linear relationship was shown between the mesothelioma incidence/mortality rate and the preceding per capita asbestos consumption rate with the Spearman correlation coefficient at $0.70(p=0.03)$, and $R^{2}$-value at $66 \%$. However, the data-point for Japan was situated apart from the linear relationship due to the lower mesothelioma mortality rate, and when combined with other Western countries, the significant relationship diminished. It is possible that the asbestos consumption curve for Japan in past years lagged behind that for the Western countries and the cumulative exposure effect has not yet reached the level that can be expected from other Western countries.
\end{abstract}

(J Occup Health 1999; 41: 8-11)

Key words: Mesothelioma, Asbestos, Ecological study, National statistics

Received July 7, 1998; Accepted Oct 30, 1998

Correspondence to: $K$. Takahashi, Department of Environmental Epidemiology, Institute of Industrial Ecological Sciences, University of Occupational and Environmental Health, Orio, Yahatanishiku, Kitakyushu City 807-8555, Japan
The International Expert Meeting on Asbestos, Asbestosis and Cancer was convened in Helsinki in January, 1997 to discuss disorders of the lung and pleura in association with asbestos and to agree upon state-ofthe-art criteria for their diagnosis and attribution with respect to asbestos. The meeting was attended by 19 internationally recognized experts in relevant disciplines from eight countries not producing asbestos ${ }^{1,2)}$. Among the many fruits of the meeting was the recognition of the importance of an ecological perspective, in particular the observation of similar patterns in the asbestos consumption curve followed by a growing trend in asbestos-related cancers in many countries. The term "the global asbestos epidemic" was used to characterize asbestos-related health issues on a global scale seen today and expected in the foreseeable future ${ }^{3)}$. It was in this context that the mortality/incidence rates of mesothelioma in contrast to asbestos consumption were tabulated for ten Western countries ${ }^{4}$. The justification for such a procedure is that mesothelioma is closely related to asbestos exposure, and mesothelioma mortality can be taken as an index of past exposure to asbestos in the population ${ }^{S \text {. }}$.

Japan is one of the largest consumer countries of asbestos ${ }^{6)}$ and although the country was represented at the meeting, its data on mesothelioma incidence/mortality were not available and were therefore precluded from the cross-country comparison. Since the meeting, national mortality statistics on mesothelioma became available for the first time in Japan due to the adoption of the Tenth Revision of the International Statistical Classification of Diseases and Related Health Problems, or ICD-107). This provided a unique opportunity to combine and compare data for Japan with those for Western countries and to further focus on the variation in data between countries from the viewpoint of geographical correlation. Neither assessment had been done in the earlier effort. The objective of the present study was therefore to evaluate 
the ecological relationship between mesothelioma incidence/mortality and asbestos consumption in countries including Japan where relevant data were available.

\section{Methods}

In the initial tabulation of ten Western countries, national incidence/mortality rates were calculated from the most recent figures for malignant mesothelioma cases/ deaths for the population over 15 years of age (/one million/year). The per capita asbestos consumption was calculated for all types of asbestos consumed approximately $10-25$ years ago as a rate for the national population $(\mathrm{kg} / \mathrm{capita} / \mathrm{ye}$ ar). It should be noted that currently there is no single information source designated to make cross-country comparisons of current mesothelioma incidence/mortality rates or asbestos consumption figures for the past. Data were therefore compiled from fourteen different information sources, all of which were scientific papers but with independent objectives. The tabulated figures and corresponding information sources are given elsewhere ${ }^{4)}$.

Japan's mortality rate of mesothelioma for the year 1995 was calculated by the same procedure based on the official vital statistics of Japan ${ }^{8}$. Japan's asbestos consumption figure was taken from the national import statistics" for the year 1974.
As the ecological relationship, Spearman's correlation coefficient and a regression formula was calculated by SAS software on a personal computer.

\section{Results}

As shown in the figure, recent mesothelioma incidences ranged between 5 (Japan) and 25 (Australia) with a median of 15 per one million per year (United States). The per capita asbestos consumption rate ranged between 1.9 (Norway) and 4.4 (Australia) with a median of 2.6 $\mathrm{kg}$ per capita per year (Italy).

Among the ten Western countries there is a clear linear relationship between the mesothelioma incidence/ mortality rate and per capita asbestos consumption rate lagged for 10-25 years. The Spearman correlation coefficient of the two variables among the ten Western countries is $r=0.70(p=0.03)$. Further, the per capita asbestos consumption rate $(x)$ can be regressed on the mesothelioma incidence rate $(y)$ by the formula: $y=6.08 x$ $+0.55(\mathrm{p}=0.004)$, and $\mathrm{R}^{2}=0.66$ (adjusted $-\mathrm{R}^{2}=0.62$ ), indicating that $66 \%$ ( $62 \%$ when adjusted) of the variation of $y$ can be explained by $x$.

The data-point for Japan is situated apart from the linear relationship. The per capita asbestos consumption rate of $3.1 \mathrm{~kg}$ per capita per year $(350,000$ metric tons and 111.94 million people of all ages for the year 1974) is well within the range for Western countries (i.e., one rank

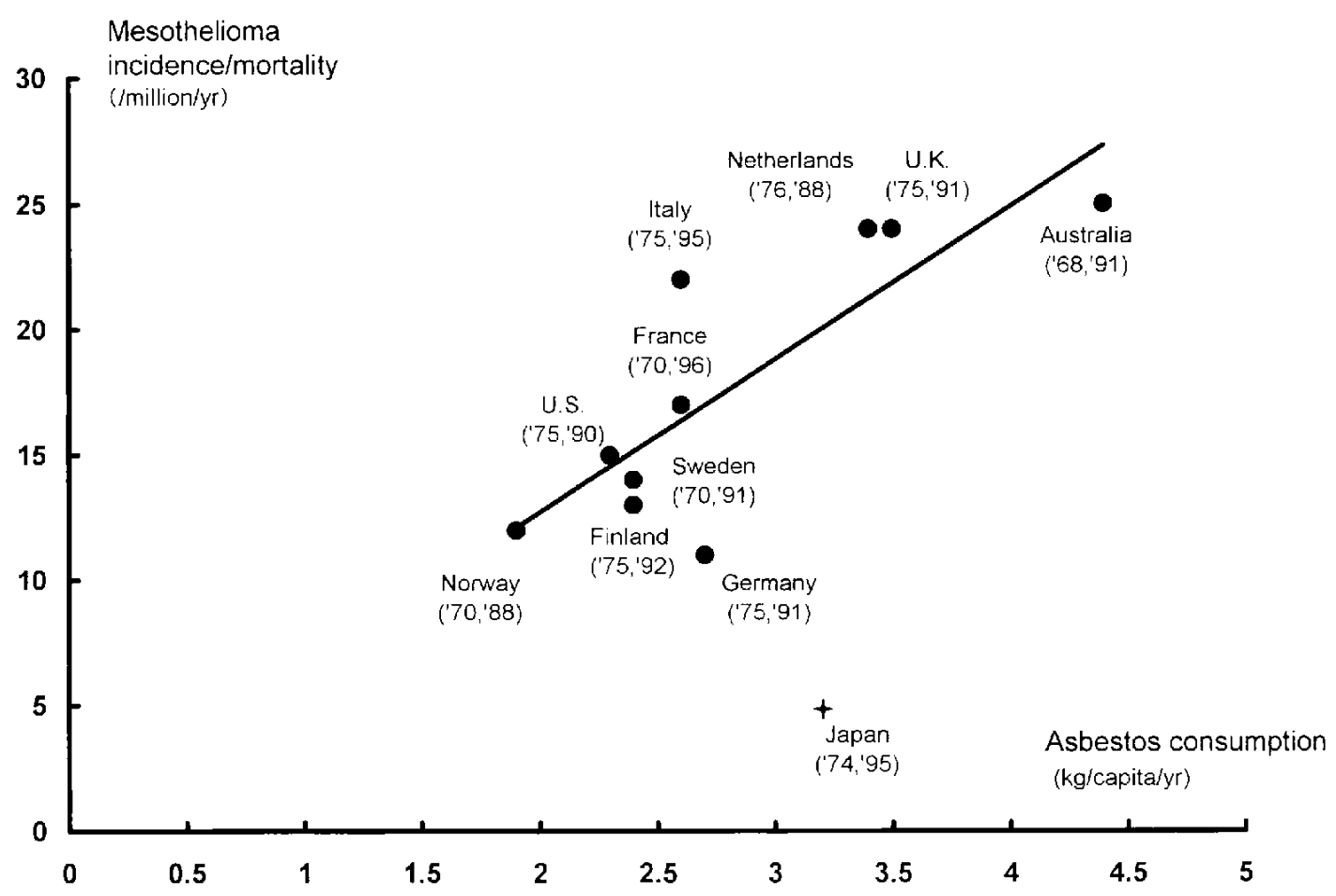

Figure Geographical correlation between per capita asbestos consumption and mesothelioma incidence/ mortality (Figures in brackets indicate reported year of asbestos consumption and mesothelioma incidence/mortality, respectively). 
above the median value), whereas the mesothelioma incidence rate of 5 per one million ( 500 deaths and 105.42 million people over 15 years of age for the year 1995) is much lower than that of Western countries (i.e., less than half the minimum value). The linear relationship between the two variables diminished when Japan's data were combined with those of Western countries, with the Spearman correlation coefficient of the two variables reducing to $r=0.52(p=0.10)$.

\section{Discussion}

This is the first report examining the ecological relationship between mesothelioma incidence/mortality and asbestos consumption at the national level in a number of countries. The highly specific relationship between asbestos exposure and mesothelioma development, well recognized at the individual level, is likely to reinforce the demonstration of an ecological relationship at regional levels. The geographical relationship shown in this study reflects another type of ecological relationship, i.e., the time-trend curves of asbestos consumption followed by its health consequences in each country. Although ecological studies are often discredited other than for hypothesisgenerating purposes, their strengths and advantages in providing leads to public health measures should not be underestimated $^{10)}$. This is particularly true in view of the spread and shift of asbestos consumption towards developing countries and countries of transition with limited mechanisms for prevention and control ${ }^{33}$.

In the present study, a linear ecological relationship was apparent between per capita asbestos consumption and the time-lagged mesothelioma incidence/mortality rates for the ten Western countries. Availability of data was the sole reason for the selection of the countries. Similarly, the choice of length for lag time was constrained by the scarce information on asbestos consumption for earlier years. Such logistic constraints should have decreased, rather than increased, the potential bias of the data incorporated into the study. The major limitation of the analysis is the lack of account for national differences in population characteristics, mesothelioma diagnoses and registration as well as in asbestos fibres or products used by the asbestos industry and various endusers. For the ten Western countries, the regional variation in mesothelioma incidence/mortality is significantly associated with that of per capita asbestos consumption in the past. Furthermore, the regression formula suggests that, i) each $\mathrm{kg} /$ capita/yr increase in asbestos consumption is related to an increase in a mesothelioma incidence of 6 per one million/year; ii) $66 \%$ of the variation in mesothelioma incidence/mortality rate can be explained by the per capita consumption of asbestos; iii) 0.5 mesothelioma case per million per yr may occur independent of the per capita consumption of asbestos.
The data for Japan were positioned apart from the linear relationship due to the exceptionally low mesothelioma mortality rate in comparison with the ten Western countries. We believe there is a plausible explanation for this. The disparity can largely be explained by the fact that the asbestos consumption curve of Japan in past years paralleled the historical "catch-up" pattern of the industrial growth curve characteristic of the country, i.e., lagging behind Western countries followed by a rapid increase to eventually catch up or exceed the Western level. Because the time lag between the onset of asbestos exposure and mesothelioma occurrence may exceed 40 years, the later growth of asbestos consumption can be reflected in the comparatively low level of mesothelioma mortality witnessed today, but such a speculation is qualitative and requires verification. Morinaga and other Japanese researchers have made similar speculations ${ }^{\text {(1-13) }}$ based on regional data. The current national mortality rate of 5 per one million per year is, in fact, considerably higher than the estimated rates for Japan reported in the past $^{14,15)}$. It is possible that the data for Japan will shift towards the regression line represented by Western countries as the cumulative exposure effect becomes apparent. This will add to the importance of closely monitoring the incidence/mortality data based on the accurate diagnosis and registration of mesothelioma cases in Japan.

The quantity, quality and comparability of data combined from various information sources limit inferences. Nevertheless, interesting leads were obtained to justify the planning of an ecological study with an extended and specific design. The subsequent study design should cover a longer period and as many countries and regions as possible in a comparable format. International agreement on the terminology and criteria for including data such as diagnosis, asbestos fibre type and age-adjustment will ultimately determine the value and validity of the ecological approach. Moreover, the acquisition of comparable data and the use of experience in different countries will promote research and discussion aimed at the quantitative evaluation of the global asbestos epidemic. From the ethical point of view, it is imperative for the medical profession to contribute to the prevention, detection, diagnosis of, and just compensation for asbestos-related diseases ${ }^{16,17)}$.

\section{References}

1) Consensus report: Asbestos, asbestosis, and cancer: the Helsinki criteria for diagnosis and attribution. Scand $\mathbf{J}$ Work Environ Health 1997; 23: 311-316.

2) Tossavainen A, Huuskonen MS, Rantanen J. Preface. In: Tossavainen A, Huuskonen MS, Rantanen J, eds. Proceedings of an International Expert Meeting on Asbestos, Asbestosis, and Cancer. Helsinki: Finnish Institute of Occupational Health, 1997: i-ii.

3) Rantanen J. Global asbestos epidemic-is it over? In: Tossayainen A, Huuskonen MS, Rantanen J, eds. 
Proceedings of an International Expert Meeting on Asbestos, Asbestosis, and Cancer. Helsinki: Finnish Institute of Occupational Health, 1997: 1-4.

4) Tossavainen A. Asbestos, asbestosis and cancer. In: Tossavainen A, Huuskonen MS, Rantanen J, eds. Proceedings of an International Expert Meeting on Asbestos, Asbestosis, and Cancer. Helsinki: Finnish Institute of Occupational Health, 1997: 8-20.

5) Peto J, Hodgson JT, Matthews FE, Jones JR. Continuing increase in mesothelioma mortality in Britain. Lancet 1995; 345: 535-539.

6) Takahashi K, Tsuda T, Higashi T. Asbestos research issues in Japan. In: Peters GA and Peters BJ, eds. Sourcebook on Asbestos Diseases: Causation, Diagnosis, Therapy, and Related Aspects. Volume 17, Pathology, Immunology, and Gene Therapy. Charlottesville: Lexis Law Publishing, 1998: 295-310.

7) World Health Organization. International Statistical Classification of Diseases and Related Health Problems, Tenth Revision. Geneva: World Health Organization, 1992.

8) Ministry of Health and Welfare. Table 1-1. Deaths, by causes (the list of three character categories), sex and age: Japan, 1995-con. In: Vital Statistics of Japan 1995. Tokyo: Statistics and Information Department, Minister's Secretariat, Ministry of Health and Welfare, 1998: 116-339.

9) Environment Agency, Japan. Review of Air Pollutants: All about Asbestos and Zeolite (in Japanese). Kawasaki: Japan Environment Hygiene Center, 1987: 16.
10) Pearce N. Traditional epidemiology, modern epidemiology, and public health. Am J Public Health 1996; 86: 678-683.

11) Morinaga $K$, Yokoyama K, Sera $Y$, Malignant pleural mesothelioma. Gendai Iryo 1984; 16: 286-293 (in Japanese).

12) Sera $Y$, Yokoyama K, Morinaga K. Lung cancer among asbestos workers. Roudou no Kagaku 1981; 36 (11): 12-19 (in Japanese).

13) Baba K. Indications of an increase of occupational pleural mesothelioma in Japan. Journal of UOEH (Sangyo lka Daigaku Zasshi) 1983; 5 (1): 3-15.

14) Morinaga K, Hara I, Miura T, Sera Y. Malignant mesothelioma and asbestos exposure. Jpn J Industrial Health (Sangyo Igaku) 1982; 24: 844 (abstract, in Japanese).

15) Morinaga K, Fujimoto I, Sera Y. Epidemiology of malignant mesothelioma in Japan. Lung Cancer (Haigan) 1987; 27 (5): 474 (71) (abstract, in Japanese).

16) International Commission on Occupational Health. International Code of Ethics for Occupational Health Professionals. International Commission on Occupational Health, 1992.

17) Rantanen J, Henderson DW. Criteria for diagnosis and attribution of asbestos diseases. In: Proceedings of the Asbestos Symposium for the Countries of Central and Eastern Europe. Helsinki: Finnish Institute of Occupational Health. People and Work-Research Reports 19. 1998; 12-18. 